\title{
BMJ Open Scheduled Prophylactic 6-Hourly IV AcetaminopheN to Prevent Postoperative Delirium in Older CaRdiac SurgicAl Patients (PANDORA): protocol for a multicentre randomised controlled trial
}

\author{
Tanvi Khera (D) , ${ }^{1}$ Pooja A Mathur, ${ }^{1}$ Valerie M Banner-Goodspeed (D) , \\ Shilpa Narayanan, ${ }^{1}$ Marie Mcgourty, ${ }^{1}$ Lauren Kelly, ${ }^{1}$ Kerry Palihnich, ${ }^{2}$ \\ Lena Novack (1) , ${ }^{3,4}$ Roger Davis, ${ }^{2}$ Daniel Talmor, ${ }^{1}$ Edward R Marcantonio, ${ }^{2}$ \\ Balachundhar Subramaniam (D) ${ }^{1}$
}

To cite: Khera T, Mathur PA, Banner-Goodspeed VM, et al. Scheduled Prophylactic 6-Hourly IV AcetaminopheN to Prevent Postoperative Delirium in Older CaRdiac SurgicAl Patients (PANDORA): protocol for a multicentre randomised controlled trial. BMJ Open 2021;11:e044346. doi:10.1136/ bmjopen-2020-044346

- Prepublication history for this paper is available online. To view these files, please visit the journal online (http://dx.doi. org/10.1136/bmjopen-2020044346).

ERM and BS are joint senior authors.

Received 31 August 2020 Revised 03 December 2020 Accepted 08 February 2021

Check for updates

(C) Author(s) (or their employer(s)) 2021. Re-use permitted under CC BY-NC. No commercial re-use. See rights and permissions. Published by BMJ.

For numbered affiliations see end of article.

Correspondence to

Dr Balachundhar Subramaniam; bsubrama@bidmc.harvard.edu

\section{ABSTRACT}

Introduction Postoperative delirium is common among older cardiac surgery patients. Often difficult to predict and address prophylactically, delirium complicates the postoperative course by increasing morbidity and mortality as well as prolonging both hospital and intensive care unit (ICU) lengths of stay. Based on our pilot trial, we intend to study the effect of scheduled 6-hourly acetaminophen administration for 48 hours post-cardiac surgery with cardiopulmonary bypass (CPB) on the incidence of inhospital delirium and long-term neurocognitive outcomes. Additionally, effect on duration and severity of delirium, rescue analgesic consumption, acute and chronic pain scores and lengths of hospital and ICU stay will also be explored.

Methods and analysis This multicentre, randomised, placebo-controlled, quadruple-blinded trial will include 900 older ( $>60$ years) cardiac surgical patients requiring CPB Patients meeting the inclusion criteria and not meeting any exclusion criteria will be enrolled at seven centres across the USA with Beth Israel Deaconess Medical Center (BIDMC), Boston, as the central coordinating centre. Additional sites may be included to broaden or speed accrual. The primary outcome measure is the incidence of in-hospital delirium till day 30 . Secondary outcomes include the duration and severity of in-hospital delirium, hospital and ICU lengths of stay, postoperative pain scores, postoperative rescue analgesic consumption, postoperative cognitive function and chronic sternal pain. Creation of a biorepository and the use of intraoperativeblinded electroencephalogram (EEG) and cerebral oximetry data will support exploratory endpoints to determine mechanistic predictors of postoperative delirium. Ethics and dissemination This trial is approved and centrally facilitated by the Institutional Review Board at BIDMC. An independent Data Safety and Monitoring Board is responsible for maintaining safety oversight. Protocol \# 2019P00075, V.1.4 (dated 20 0ctober 2020).

Trial registration number NCT04093219.

\section{Strengths and limitations of this study}

This study will provide generalisable evidence for prophylactic efficacy of acetaminophen in reducing the incidence of postoperative delirium in older cardiac surgical patients.

- This study will provide insight into the impact of acetaminophen on long-term trajectories of cognitive dysfunction, functional status and chronic pain after cardiac surgery.

- The proposed secondary analysis of intraoperativeblinded EEG and cerebral oximetry will help evaluate the role of intraoperative variability as a causate for postoperative delirium.

- The plasma and DNA samples collected will serve as a biorepository to aid further research to identify biomarkers that serve as early predictors of delirium in high-risk patients.

- Despite well-planned cohort retention strategies, maintaining cohorts for prolonged periods can be challenging.

\section{INTRODUCTION}

Delirium is characterised by an acute onset decline in attention and cognitive function, which may have a fluctuating course. Postoperative delirium is common in the older cardiac surgical population, with an incidence as high

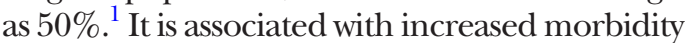
and mortality, prolonged hospital and intensive care unit (ICU) lengths of stay, long-term cognitive dysfunction and functional decline. ${ }^{1}$ Postoperative cognitive dysfunction, now termed postoperative neurocognitive disorder, is defined as a significant deterioration of cognitive function following surgery and is especially 


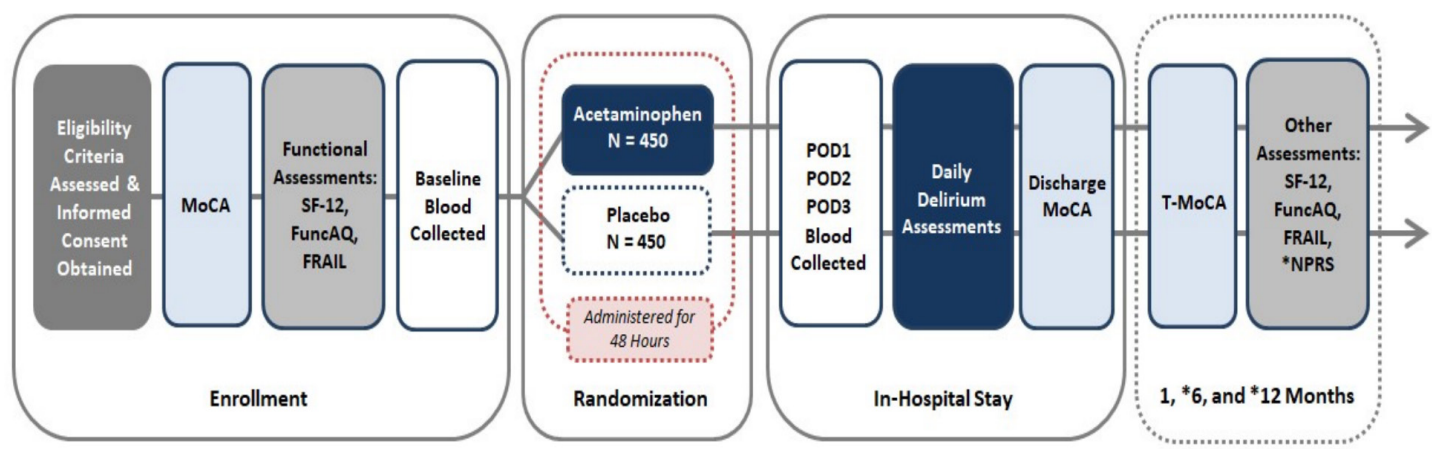

Figure 1 Study schema. FRAIL, 5-item Frailty Questionnaire; FuncAQ, Functional Assessment Questionnaire; MoCA, Montreal Cognitive Assessment; NPRS, Numeric Pain Rating Scale; POD: postoperative day; SF-12, Short Form 12; T-MoCA, Telephonic MoCA.

concerning for the cardiac surgical population..$^{2-5}$ Postoperative delirium is known to increase cognitive dysfunction for 6-12 months after cardiac surgery. ${ }^{267}$ The risk factors for delirium are multifactorial, including preoperative cognitive dysfunction, intraoperative anaesthetics, intraoperative haemodynamics, duration and complexity of the surgery, use of cardiopulmonary bypass (CPB), postoperative pain and use of postoperative opioids. ${ }^{89}$ There is a growing body of evidence that suggests the role of neuroinflammatory mediators in the occurrence of postoperative delirium which either cross the blood-brain barrier or are centrally produced in the setting of systemic inflammation. ${ }^{10}$ These neuroinflammatory pathways may be potentiated with the use of $\mathrm{CPB}$ and ischaemia-reperfusion injuries during cardiac surgeries. ${ }^{11}$ Suboptimal pain management and excessive use of opioids both increase the risk of postoperative delirium, posing a challenge for the treating physicians. Since we do not understand the aetiology of postoperative delirium, there are currently no targeted prophylactic modalities for postoperative delirium. Some prophylactic medications, such as antipsychotics, sedatives and dexmedetomidine, used previously have uncertain efficacy, though a recent meta-analysis concluded that antipsychotics were not effective for either prevention or treatment of delirium. ${ }^{12-16}$

One potential intervention to prevent delirium is use of postoperative non-opioid analgesics. Due to growing concerns of opioid misuse in the perioperative period, the current practice trends towards the use of multimodal analgesic regimens. ${ }^{17}$ Multimodal analgesia widely relies on the use of non-steroidal anti-inflammatory drugs (NSAIDs) and acetaminophen as an adjuvant in the standard analgesic protocol. ${ }^{1819}$ NSAIDs are limited by their unique concerns in the setting of cardiac surgical patients. There is an increased risk of bleeding and renal failure, which can be potentiated with the use of NSAIDs. ${ }^{20}$ Acetaminophen is a centrally acting, non-opioid analgesic modality. ${ }^{21}{ }^{22}$ The bioavailability of intravenous acetaminophen is higher and more reliable as compared with an equivalent dose of oral formulation as it bypasses the variable first-pass metabolism. ${ }^{23-25}$ This characteristic provides a highly predictable and superior pharmacokinetic and pharmacodynamic profile to the intravenous formulations, particularly in the first few days after surgery. Current evidence suggests that acetaminophen has anti-inflammatory properties when used intravenously in the postoperative period. ${ }^{26}$

In the recent DEXACET trial, we documented a significant reduction in the incidence of delirium in cardiac surgical patients who received scheduled 6-hourly acetaminophen in the postoperative period. ${ }^{27}$ This pilot study was a prospective, randomised, placebo-controlled trial with factorial group assignments carried out at a single centre. We compared the incidence of delirium, lengths of hospital and ICU stay and long-term cognitive decline in 120 patients undergoing cardiac surgery using a predefined analgesic and sedative regime. This included a comparison between groups receiving either acetaminophen or placebo as analgesics with either dexmedetomidine or propofol as the sedative in the postoperative period. The analgesic regime used intravenous acetaminophen, given as eight doses, 6hours apart in the first 48 hours following surgery. The primary outcome was incidence of in-hospital delirium using the Confusion Assessment Method (CAM) ${ }^{28}$ and the secondary outcomes included delirium duration, cognitive decline, breakthrough opioid analgesia requirement in the first 48 hours and the ICU and hospital lengths of stay. There was a significantly decreased incidence of in-hospital delirium in patients randomised to postoperative acetaminophen versus placebo ( $10 \%$ vs $28 \%$ placebo; difference, $-18 \%$; $\mathrm{p}=0.01$ ); there was also a significant reduction in the three prespecified secondary outcomes-duration of delirium, ICU length of stay and use of breakthrough analgesia (measured in morphine equivalents) in these patients. The baseline and following cognitive assessments were carried out using the Montreal Cognitive Assessment (MoCA), delirium assessment was performed using the CAM, functional status was assessed using the Activities of Daily Living (ADL) and Instrumental ADL (IADL) scales and mood was assessed using the Geriatric Depression Scale (GDS). ${ }^{29}{ }^{30}$ The biggest challenge faced during this pilot study was cohort retention throughout the follow-up period, which may have impacted interpretation of long-term outcomes.

Data from our pilot study laid the foundation for this multicentre trial. The objective of the Scheduled Prophylactic 6-Hourly IV AcetaminopheN to Prevent Postoperative Delirium in Older CaRdiac SurgicAl Patients (PANDORA) clinical trial is to study the impact of 6-hourly intravenous 


\begin{tabular}{|c|c|c|c|c|c|c|c|c|c|}
\hline & \multirow{2}{*}{$\begin{array}{c}\text { Pre-Hospital } \\
\text { Baseline }\end{array}$} & \multicolumn{5}{|c|}{ In-Hospital } & \multicolumn{3}{|c|}{ Postoperative Follow Up } \\
\hline & & $\begin{array}{c}\text { Study Day } 0^{3} \\
\text { (Day of Surgery) }\end{array}$ & $\begin{array}{l}\text { Study } \\
\text { Day } 1\end{array}$ & Study Day 2 & $\begin{array}{l}\text { Study Day } 3 \text { to } \\
\text { Discharge }\end{array}$ & $\begin{array}{c}\text { Hospital } \\
\text { Discharge / } \\
\text { Day } 30^{\mathrm{b}}\end{array}$ & $\begin{array}{c}1 \\
\text { Month }\end{array}$ & 6 Months & $\begin{array}{c}12 \\
\text { Months }\end{array}$ \\
\hline \multicolumn{10}{|l|}{ Eligibility } \\
\hline Screening & $\mathrm{x}$ & & & & & & & & \\
\hline Entry Criteria & $\mathrm{x}$ & & & & & & & & \\
\hline Informed Consent & $\mathrm{x}$ & & & & & & & & \\
\hline Liver Function Tests & $\mathbf{x}^{\mathrm{c}}$ & & & $\mathbf{X}^{\mathrm{k}}$ & & & & & \\
\hline Demographics & $x$ & & & & & & & & \\
\hline Medical History & $x$ & & & & & & & & \\
\hline \multicolumn{10}{|l|}{\begin{tabular}{|l} 
Study Medication \\
\end{tabular}} \\
\hline Randomization & & $\mathrm{x}$ & & & & & & & \\
\hline Study Drug Administration $^{d}$ & & $x$ & $\mathrm{x}$ & $\mathrm{x}$ & & & & & \\
\hline \multicolumn{10}{|l|}{ Data Collection } \\
\hline MoCA & $x$ & & & & & $\mathrm{x}$ & & & \\
\hline DSI & $\mathrm{x}$ & & $\mathrm{x}$ & $\mathrm{x}$ & $\mathrm{x}$ & $\mathrm{x}$ & $\mathrm{x}$ & $\mathrm{x}$ & $\mathrm{x}$ \\
\hline GDS & $\mathrm{x}$ & & & & & & $\mathrm{x}$ & $\mathrm{x}$ & $\mathrm{x}$ \\
\hline CAM $* /$ CAM-ICU & $\mathrm{x}$ & & $x$ & $\mathrm{x}$ & $\mathrm{x}$ & $x$ & & & \\
\hline T-MoCA & & & & & & & $x$ & $x$ & 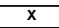 \\
\hline SF-12 & $x$ & & & & & & $x$ & $x$ & $x$ \\
\hline FuncAQ & $x$ & & & & & & $x$ & $x$ & $\mathrm{x}$ \\
\hline FRAIL & $x$ & & & & & & $x$ & $x$ & $x$ \\
\hline Collection of Clinical Dataf & & $\mathrm{x}$ & $\mathrm{x}$ & $\mathrm{x}$ & $\mathrm{x}$ & $\mathrm{x}$ & & & \\
\hline Pain Scores 9 & & & $\bar{x}$ & $x$ & $x$ & $x$ & & $\mathrm{x}$ & $\mathrm{x}$ \\
\hline Sample Collection ${ }^{\mathrm{h}}$ & $\mathrm{x}$ & & $x$ & $x$ & & & & & \\
\hline $\begin{array}{l}\text { Measurement of Rescue } \\
\text { Analgesics }\end{array}$ & & & & & & $\mathrm{X}$ & & & \\
\hline Major Postsurgical Outcomes & & & & & & & $\mathbf{x}$ & & \\
\hline \multicolumn{10}{|l|}{ Monitoring } \\
\hline Safety Monitoring & & $\bar{x}$ & $\mathrm{x}$ & $\bar{x}$ & $\overline{\mathbf{X}}$ & & & & \\
\hline
\end{tabular}

Figure 2 Standard Protocol Items: Recommendations for Interventional Trials figure. The schedule of enrolment, interventions and assessment in the study. (a) Study day 0 , day of surgery; study day 1, postoperative day 1, etc. (b) Whichever comes first. (c) Use clinically available liver function tests (LFTs) (Aspartate transaminase (AST), Alanine transaminase (ALT), Alkaline phosphatase (ALP)) within 30 days, otherwise place order for research. (d) Intravenous acetaminophen/placebo administered in 6-hourly intervals for eight doses total. (e) In order to ensure appropriate identification, the CAM is assessed in-hospital until discharge with a standard daily cognitive assessment including three-object registration, DOWB, MOYB, recall, digits forward, digits backwards and orientation. (f) Clinically available data include medication administration, intraoperative blood pressures and routine labs; intraoperative sedation data. (g) Pain scores will be collected three times daily; NPRS at 6 and 12 months. (h) Sample no.1 (baseline sample) to be collected after enrolment (at the time of the preoperative study assessments or on day of surgery), prior to study drug administration and sample no. 2 (postoperative sample) to be drawn on postoperative day 1 and sample no. 3 on postoperative day2, preferably in the afternoon when patient is in the ICU. (i) Major postsurgical outcomes obtained from Society of Thoracic Surgeons database 1 month from discharge: reoperation (bleeding), deep sternal infection, stroke, pneumonia, renal failure, atrial fibrillation and death. (j) Monitoring for all adverse events will take place daily during the patient's hospital stay for a maximum of 5 days from the final dose of study medication. $(k)$ LFTs will be ordered on the morning of POD 2. CAM, Confusion Assessment Method; DOWB, days of the week backwards; DSI, delirium symptom interview; FRAIL, 5-item Frailty Questionnaire; FuncAQ, Functional Assessment Questionnaire; GDS, Geriatric Depression Scale; MoCA, Montreal Cognitive Assessment; MOYB, months of the year backwards; NPRS, Numeric Pain Rating Scale; POD, postoperative day; SF12, Short Form 12; T-MoCA, Telephonic MoCA.

acetaminophen administration for 48 hours on the incidence, duration and severity of delirium, acute and chronic pain, hospital and ICU lengths of stay, postoperative rescue analgesic requirement and the long-term trajectory of cognitive and functional status. This study cohort will include 900 older cardiac surgical patients requiring CPB from seven sites across the USA.

\section{METHODS AND ANALYSES \\ Study design}

This is a multicentre, randomised, placebo-controlled, quadruple-blinded trial designed to study the effect of postoperative scheduled 6-hourly acetaminophen administration and incidence of in-hospital delirium and longterm neurocognitive outcomes. Subjects who will meet the inclusion criteria and no exclusion criteria will be recruited after obtaining written informed consent. Recruitment will take place at seven sites across the USA: Yale-New Haven Medical Center; University of Pittsburgh Medical Center, Shadyside; University of Pittsburgh Medical Center, Presbyterian; University of Alabama; Massachusetts General Hospital; Brigham and Women's Hospital and Beth Israel Deaconess Medical Center (BIDMC).
The process of consenting will be carried out by a study physician. The study schema is presented in figure 1. Figure 2, the Standard Protocol Items: Recommendations for Interventional Trials, demonstrates the schedule of enrolment, interventions and assessments to be used in the trial.

\section{Study registration}

This trial is approved by the Committee on Clinical Investigations (CCI), Institutional Review Board (IRB) at BIDMC, Boston, which serves as the single IRB (sIRB) for this study. All other participating institutions grant human subjects approval via reliance agreements with the sIRB. This trial is prospectively registered with the US National Institutes of Health on ClinicalTrials.gov as of 17 September 2019. The study began recruiting in August 2020 and expected to be open for recruitment for the next 4 years (October 2024).

\section{Inclusion and exclusion criteria}

We will include 900 older ( $>60$ years) cardiac surgical patients undergoing coronary artery bypass grafting with or without a valve or isolated valve surgery requiring complete $\mathrm{CPB}$ in this trial (with medial sternotomy approach). Patients with preoperative left ventricular ejection fraction $<30 \%$, emergent procedures, isolated aortic 
Table 1 Inclusion and exclusion criteria with rationale

\begin{tabular}{|c|c|}
\hline Inclusion criteria & Rationale \\
\hline $\begin{array}{l}\text { Undergoing cardiac surgery (CABG with or without valve, isolated } \\
\text { valve surgery) requiring CPB }\end{array}$ & $\begin{array}{l}\text { Cardiopulmonary bypass surgeries are associated with an increased } \\
\text { risk for delirium }\end{array}$ \\
\hline Exclusion criteria & Rationale \\
\hline Preoperative LVEF less than $30 \%$ & $\begin{array}{l}\text { Inability to adequately assess outcome (expected to stay intubated for } \\
\text { longer period of time) }\end{array}$ \\
\hline Aortic surgery & $\begin{array}{l}\text { Inability to adequately assess outcome (expected to stay intubated for } \\
\text { longer period of time) }\end{array}$ \\
\hline $\begin{array}{l}\text { Liver dysfunction (ALT or AST > } 3 \text { times the upper limit of local normal; } \\
\text { all patients will have a baseline liver function test information or history } \\
\text { and examination suggestive of jaundice or both) }\end{array}$ & Intervention contraindicated \\
\hline Any history of alcohol withdrawal or delirium tremens & Inability to adequately assess outcome \\
\hline Active delirium at baseline & Inability to adequately assess incidence and define baseline \\
\hline Non-English speaking & $\begin{array}{l}\text { Inability to administer cognitive assessments, which have been } \\
\text { extensively validated in English }\end{array}$ \\
\hline Physician refusal & Inability to administer intervention per clinician preference \\
\hline Prisoner & Population requires additional OHRP protections \\
\hline
\end{tabular}

CABG, coronary artery bypass grafting; CPB, cardiopulmonary bypass; LVEF, left ventricular ejection fraction; OHRP, Office for Human Research Protections.

surgeries, liver dysfunction (Alanine transaminase (ALT) or Aspartate transaminase (AST) $>3$ times the upper limit of local normal or either history or examination suggestive of jaundice), hypersensitivity to the study drug, history of alcohol abuse within the past year ( $\geq 5$ drinks per day for men and $\geq 4$ drinks per day for women) or history of alcohol withdrawal or delirium tremens, evidence of delirium at baseline (as identified at the preoperative baseline CAM assessment), clinician disapproval for participation, English language limitation or prisoner status will be excluded from the study. A full listing of trial exclusions and rationale is displayed in table 1 .

\section{Randomisation and study intervention}

Randomisation and blinding

After obtaining written informed consent, participants will be randomised to receive either $1 \mathrm{~g}$ of intravenous acetaminophen or placebo in a 1:1 allocation ratio. Permuted block randomisation of variable block sizes two, four and six will be utilised, stratified by institution. Computer-generated randomisation schemas will be made available at site-specific designated research pharmacies, which will be responsible for preparing and dispensing the study drug as per the allocated group. Acetaminophen and placebo will be dispensed in identically packaged intravenous bags to ensure blinding. The participant, clinical care providers, investigator and outcomes assessor are blinded to the group assignment, making this a quadruple-blinded trial.
Study data collection protocol

Preoperative and intraoperative management will be at the discretion of the treating physicians as per the usual standard of care. Preoperatively, consented patients will undergo baseline neurocognitive and functional assessments including the delirium symptom interview (DSI), GDS, MoCA, Functional Assessment Questionnaire (FuncAQ), 5-item Frailty Questionnaire (FRAIL) and Short Form (SF-12). ${ }^{29-34}$ The presence or absence of delirium will be determined using the CAM diagnostic algorithm, which will be scored by the interviewer based on the above assessments; the presence of preoperative delirium is exclusionary. These assessments will be followed by a baseline blood sample collection, which will be used to assess baseline liver function (if not already obtained as part of clinical care) and also stored in the study biorepository. Assuming all inclusion criteria are met and there are no exclusions, the patient will be randomised as described above.

Intraoperatively, the patient will undergo EEG monitoring and cerebral oximetry using Sedline Root monitor. The data obtained intraoperatively will be used to support the secondary outcome and will not impact the anaesthetic management of the patients. The medication regime of either $1 \mathrm{~g}$ of intravenous acetaminophen or placebo will commence within an hour of transfer to the ICU from the operating room. The study medication will be repeated for a total of 8 doses, each 6 hours apart for the first 48 hours. For patients $<50 \mathrm{~kg}$, the intravenous 


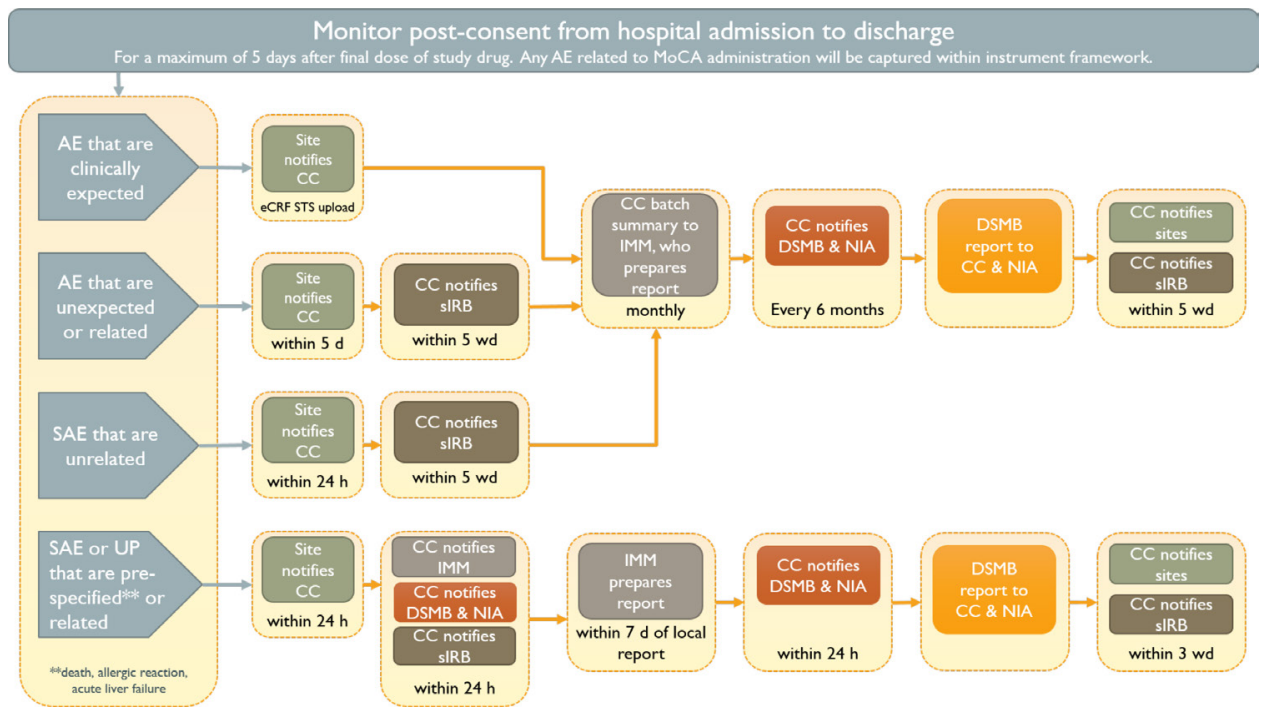

Figure 3 AE reporting schema. AE, adverse event; CC, coordinating centre; d, days; DSMB, Data Safety and Monitoring Board; eCRF, electronic Case Report Forms; h, hours; IMM, Independent Medical Monitor; MoCA, Montreal Cognitive Assessment; NIA, National Institute of Ageing; SAE, Serious Adverse Events; sIRB, Single Institutional Review Board; STS, Society for Thoracic Surgeons; UP, unanticipated problem; wd, working days.

acetaminophen dose will be adjusted to $15 \mathrm{mg} / \mathrm{kg}$ every 6 hours up to a total of $75 \mathrm{mg} / \mathrm{kg} /$ day. The extubation protocols and ambulation goals will be guided by the usual standard of care as defined by enhanced recovery after surgery protocol. The cardiovascular ICU's routine extubation criteria include ensuring stable haemodynamics, complete neuromuscular blockade reversal and meeting required pulmonary criteria prior to discontinuing ventilatory support. Similarly, standard clinical ambulation protocols will be followed, which include encouraging patients to move out of bed on postoperative day (POD) 1 and ambulate in the hallway (two to three times a day) on POD 2 if haemodynamically stable, ambulate in the hallway (three to four times a day) on POD 3 and use of stairs with assistance on POD 4. The study team at each site will monitor the study drug administration and adherence to protocol. Additionally, information about the patient's preoperative comorbidities, surgical procedure and sternal closure (rigid fixation or wire cerclage), hospital length of stay, intubation and extubation time, reintubation (if any), time of first mobilisation and hospital discharge will be collected from the medical record.

The study team will obtain additional blood samples on the morning of POD 1 and POD 2. The samples collected at baseline, on POD 1 and POD 2, will be fractionated into components (red cells, buffy coat and plasma). The latter two components will be frozen and shipped to the coordinating centre (CC) to be stored in PANDORA biorepository for future analyses. The plasma fraction will be utilised for biomarker analyses at the CC. The buffy coat will be stored at the CC for future genetic research. Acetaminophen levels will be obtained from the POD 2 blood sample. Liver function tests (LFTs) obtained on POD 2 (3hours after the last study dose) will be used to assess the safety of further administration of acetaminophen in any form, by ensuring that these values remain less than three times the upper limit of normal as per the local laboratory.

\section{Prohibited/restricted concomitant medications}

While the study protocol is in force, clinicians will be discouraged from using acetaminophen in any other form, to avoid drug overdose. Use of regional anaesthetic techniques and lidocaine patches can confound postoperative analgesic consumption and will be avoided. Prophylactic haloperidol for delirium in the ICU will be discouraged to ensure robust data collection for the primary outcome and because existing evidence (see above) suggests it is not effective. If the clinical care dictates the use of any of these medications (including regional techniques), it will be recorded and analysed as breakthrough or rescue medication to be analysed as a secondary outcome.

\section{Study outcome measures}

Primary endpoints

The primary outcome for the trial is the incidence of postoperative in-hospital delirium censored at day 30 of index hospital admission. The incidence of postoperative in-hospital delirium will be assessed using the CAM, which will be scored by the study team members once a day starting from POD 1 . The CAM-ICU will be used for patients who are intubated in the postoperative period. ${ }^{35}$ This daily delirium assessment will include a standardised brief cognitive assessment that tests attention, orientation and memory, followed by the DSI, which asks the patient for specific symptoms of delirium. Based on these data, trained study assessors will complete the long CAM, which assesses 10 features of delirium. From this assessment, the CAM diagnostic algorithm will be completed to determine the presence or absence of delirium on that day. If a patient scores CAM negative on three consecutive 
days following POD 2, they will be put on a skip pattern, where the assessments will then be administered on alternate days. In case a subject becomes CAM positive on a particular day, the skip cycle will reset, requiring an additional three consecutive CAM negatives to reinitiate the skip cycle. Assessments will be administered once every day up to discharge or day 30 of index hospital admission, whichever is earlier. The once daily assessment interview includes questions about the past 24 hours, such as periods of disorientation or perceptual disturbances. This information is supplemented with chart abstraction to capture instances of delirium recorded as part of clinical care.

The discharge assessment, which is similar to the baseline assessment with the exception of GDS, will be administered on or up to 2 days before the day of patient discharge.

The post-discharge follow-up assessments will be completed via telephone at 1, 6 and 12 months from the day of surgery using the Telephone-MoCA (T-MoCA), ${ }^{36}$ DSI, GDS, FuncAQ, FRAIL and CAM. In order to limit loss to follow-up, the protocol permits a window period of +14 days at 1 month, and \pm 1 month at 6 months and 12 months assessments. Figure 2 demonstrates the time points at which each study procedure must be completed.

\section{Secondary endpoints}

Secondary endpoints for this trial include the duration of delirium, delirium severity, postoperative cognitive dysfunction, analgesic consumption in the first 48 hours, hospital and ICU lengths of stay, daily pain scores and incidence of chronic sternal pain at 6 and 12 months. These endpoints will be assessed using standardised instruments: delirium severity will be measured using CAM-Severity Long Form Score, which considers all 10 features in the long CAM assessment ${ }^{37}$; postoperative cognitive dysfunction assessed at discharge using MoCA; postoperative cognitive dysfunction at 1, 6 and 12 months evaluated using T-MoCA; long-term functional and selfcare functions assessed at 1, 6 and 12 months using the SF-12, FRAIL and FuncAQ; postoperative daily pain scores assessed using the Visual Analogue Scale and persistent chronic sternal pain at 6 and 12 months evaluated using the Numerical Pain Rating Scale (NPRS). ${ }^{38}$

\section{Data collection}

Demographic data such as age, gender, ethnicity, race, body mass index, comorbidities and surgery type will be recorded from a patient's medical record. Postoperative analgesic consumption in the first 48 hours will be computed in terms of intravenous morphine equivalents in milligrams ( (intravenous hydromorphone $\times 10+$ oral hydromorphone $\times 1.6)+($ intravenous fentanyl $\times 100)+$ (intravenous morphine $\times 1+$ oral morphine $\times 0.4)+($ intravenous sufentanil $\times 1000)+($ intravenous remifentanil $\times 100)$ + (intravenous ketorolac $\times 2.5)+$ (oral oxycodone $\times 0.6)$ $+($ oral oxymorphone $\times 1.2)+($ oral codeine $\times 0.06)+$ (oral tramadol $\times 0.04)$ ) and recorded. The research staff trained for neurocognitive assessments will record daily pain scores along with the CAM data. The data about the postoperative pain scores, total opioid consumption, delirium assessments, blood tracking and shipment status will be captured in the Research Electronic Data Capture (REDCap) database. Ongoing remote monitoring by the $\mathrm{CC}$ and targeted local double data entry will be utilised to ensure high data quality. Confidentiality will be maintained at all times by using password-protected secure servers and restriction of access to only study team members.

\section{Standardised training}

The study team members across all sites will receive standardised training for neurocognitive and delirium assessments and the REDCap database entry before study initiation. Refresher and re-trainings will be provided throughout the course of the study as necessary. Additionally, training material in the form of detailed operations manuals and videos will be made available on a passwordprotected webpage on the study website (Pandora Clinical Trial. Available at: https://pandoraclinicaltrial.org/ (accessed: 12 June 2020)).

\section{Quality assurance for neurocognitive assessments}

All neurocognitive assessments will be carried out by study team members blinded to the treatment allocation. At any site, $20 \%$ of assessments will be simultaneously scored by two trained interviewers and submitted for inter-rater reliability analysis. We will target an inter-rater concordance kappa of above 0.90 for delirium presence and an intraclass correlation coefficient of over 0.80 for delirium severity. At each site, $20 \%$ of the daily delirium assessments will be video recorded and cross-checked by the neurocognitive assessment team at the CC for training and quality assurance (QA). Videos will be used only for internal study QA purposes and will be destroyed when no longer needed. Consenting to QA video recordings are voluntary and will not affect participant's enrolment in the trial or their stay in the hospital. Periodic assessments followed by re-training and educational support will be provided as necessary.

\section{Safety endpoints}

Safety endpoints will be examined for all enrolled patients who receive at least one dose of study medication and will include laboratory testing (POD 2 acetaminophen levels for the first 225 patients, POD 2 LFTs for all patients 3 hours after the last dose), expected clinical events (eg, need for additional LFTs, discontinuation of study drug, reoperation, bleeding, deep sternal infection, stroke, pneumonia, renal failure, atrial fibrillation, in-hospital mortality, arrhythmias needing treatment, acute kidney injury) and unexpected clinical events (acute liver failure, liver injury defined as ALT/AST >400 units and death during study drug regimen). All serious and unexpected adverse events will be summarised for the Data Safety and Monitoring Board (DSMB), which is a team of 
experts separate from the study investigators, responsible for maintaining safety oversight.

Trained research team members will monitor protocol adherence and report any in-hospital adverse events to the CC. Since patients in this population are undergoing high-risk surgery, it is expected that they may suffer adverse events unrelated to study participation. Thus, we will limit our adverse event monitoring to events that may be related to the study inventions or study drug administration. The $\mathrm{CC}$ will be responsible for sharing the adverse event reports with the DSMB (figure 3).

The emergency unblinding protocol will be followed if local unblinding is required for patient safety. Treatment allocation will be disclosed to the site physician after due discussion with the on-call investigator at the CC.

\section{Cohort retention strategies}

The proposed study includes an emphasis on cohort retention for robust long-term outcome assessment. To ensure this, trial team members will stay in touch with study participants by sending reminder letters before the 6-month and 12-month calls. Three preferred contact methods and a designated secondary contact will be obtained to maximise the reach. Participants will receive a reminder magnet with the study and follow-up instructions at the time of hospital discharge. Multiple phone calls may be required to reach the patient, but after multiple failed phone attempts, a hand-addressed letter will be mailed to the patient's address.

\section{Patient and public involvement}

At this time, patient advisers were not involved in the design of the study protocol considering minimal involvement of patients in the analgesic management postoperatively after cardiac surgery. The PANDORA clinical trial is registered on clinicaltrials.gov and updated at regular intervals. Results will be reported in a time-compliant manner on clinicaltrials.gov.

\section{Sample size calculation}

The sample size calculation for the proposed trial is based on the primary outcome, in-hospital delirium up to day 30 , adjusting for one planned interim analysis, which allows early stopping for efficacy. An effect size of $66 \%$ with a baseline incidence of $30 \%$ was seen in a pilot study conducted at BIDMC. ${ }^{27}$ In this trial, with the inclusion of a much broader group of patients including those with pre-existing cognitive dysfunction, it was determined that the effect size could be reduced to as low as $33 \%$. Using O'Brien Fleming's boundary to maintain the overall twosided significance level at alpha $=0.05$ and power $=0.9,402$ patients per arm will be needed to show a $33 \%$ reduction in in-hospital delirium, assuming the baseline postoperative delirium incidence is $30 \%$. To allow for roughly $10 \%$ potential dropouts (attrition will accommodate both prerandomisation withdrawals-ie, cancelled surgery-as well as postrandomisation withdrawals-ie, intraoperative change in surgery type), the trial will enrol up to 900 patients.

\section{Statistical analysis plan}

Analyses will be conducted using SAS software V.9.4 or higher (SAS Institute). Descriptive statistics of the data will be performed, pooled across all sites. Continuous data will be represented using mean $( \pm \mathrm{SD})$ or median IQR for variables not normally distributed. The ShapiroWilk test will be used to assess normality. Categorical data will be presented using frequencies and proportions.

The primary outcome of the proposed study is in-hospital delirium, defined as the occurrence of delirium on any POD during the index admission, as assessed using the CAM or CAM-ICU daily until hospital discharge for a maximum of 30 days. This variable will be defined as either present or absent for our primary analysis. The comparison of our primary outcome will be performed using Pearson's $\chi^{2}$ test. The primary analysis will be conducted on a modified intention-to-treat (ITT) basis, which includes all patients who received at least one dose of the study drug. A per-protocol analysis will also be done, where patients receiving all the eight doses of study drug will be included. Given the randomised trial design, the results of the unadjusted analysis will be reported.

Duration of delirium, defined as the total number of in-hospital PODs (censored at 30 days) in which delirium is present, and severity of delirium, evaluated both as the peak and mean CAM-severity scores over all hospital days, will be assessed between groups using a t-test or nonparametric equivalent. Inter-rater reliability testing will be performed, with percent agreement, kappa and weighted kappa reported for all CAM variables. Additional postoperative analgesic requirements will be measured as the amount of opioid (intravenous morphine or hydromorphone) and oral analgesics required for pain control in the first 48 hours postoperatively. This value will be reported as overall morphine equivalents and compared between treatment groups. Differences in overall hospital and ICU length of stay, as well as morphine equivalents, will be compared using parametric or non-parametric t-tests. Repeated measures analyses will be performed using generalised estimating equations with a robust variance to assess for differences in the worst daily pain scores on deep breathing and cough between groups. Further, a hierarchical linear regression model will be used to characterise the trajectory of T-MoCA, SF-12, FuncAQ and FRAIL scores over time. This framework was chosen because of its flexibility with the timing of patient interviews and the repeated observations in each patient over time. This will include data from patients at baseline, discharge and 1, 6 and 12 months postoperatively.

Given the randomised nature of this trial, baseline differences between groups are not anticipated. However, if baseline differences exist, multivariate model to adjust for confounders will be used. We anticipate adherence to the randomisation assignment to be high; however, it is possible that patients may not complete their course of 
the study drug. We will, therefore, perform a sensitivity analysis on a per-protocol basis, only including patients who were randomised and completed the appropriate treatment regime.

We will evaluate whether the effect of acetaminophen differs across subsets of patients defined by few targeted factors, specifically sex, age groups, treating institution, sedation regimens (dexmedetomidine vs propofol), surgery type, preoperative cognitive dysfunction (MoCA <23), history of alcohol abuse and history of chronic opioid use. We will conduct a Cochran-MantelHaenzel analysis and test for effect modification using the Breslow-Day test.

Additional exploratory analyses of the data will be conducted as deemed appropriate including the incidence of delirium in patients with preoperative neurocognitive impairment; Parkinson's and Alzheimer's disease; time to onset of delirium in patients and intraoperative hypotension and postoperative delirium.

The frequency and percentage of missing values for each variable will be collected, analysed and reported. For the proposed trial, we anticipate that the proportion of participants with missing primary endpoint values will be small $(<1 \%)$. Further, in our previous study, from which much of the preliminary data were obtained, less than $6 \%$ of all data were missing. Consequently, we do not plan to impute missing data. Any subject receiving at least one dose of the study medication will be included in the modified ITT analysis.

The primary hypothesis will be tested after primary outcome data are available for 450 participants (50\% of the target cohort). This interim analysis will be performed for efficacy and futility of the primary endpoint by the unblinded DSMB statistician, with full results reported to the DSMB. The alpha boundary value at the interim analysis will be \pm 2.78 , which corresponds to a significance level of 0.0054 . At the final analysis, the alpha boundary will be \pm 1.97 , with a corresponding significance level of 0.0492 . A review of safety data will be performed at quartiles of enrolment, without conducting formal interim analyses.

\section{ETHICS AND DISSEMINATION}

This trial was approved by the CCI IRB at BIDMC which is also the central sIRB for all the sites. Safety oversight will be under the DSMB whose members are independent from the study teams and will regularly review the safety data throughout the conduct of the study.

The results of this study will be published in a peerreviewed medical journal, as well as presented at both national and international conferences.

\section{Author affiliations}

${ }^{1}$ Anesthesia, Critical Care and Pain Medicine, Beth Israel Deaconess Medical Center, Boston, Massachusetts, USA

${ }^{2}$ Medicine, Beth Israel Deaconess Medical Center, Boston, Massachusetts, USA

${ }^{3}$ Soroka University Medical Center, Beer Sheva, Israel

${ }^{4}$ Ben-Gurion University of the Negev, Beer-Sheva, Israel

\section{Twitter Balachundhar Subramaniam @Balachundhar}

Contributors Drafting of the manuscript: BS, TK, PAM, SN, MM, LK, KP, LN, VMB-G, $\mathrm{RD}, \mathrm{DST}$ and ERM. Critical revision of the manuscript for important intellectual content: BS, VMB-G and ERM. Obtained funding: BS, DST and ERM.

Funding The work is being supported by NIH/NIA award \# 1R01AG065554. Competing interests None declared.

Patient and public involvement Patients and/or the public were not involved in the design, or conduct, or reporting, or dissemination plans of this research.

Patient consent for publication Not required.

Provenance and peer review Not commissioned; externally peer reviewed.

Open access This is an open access article distributed in accordance with the Creative Commons Attribution Non Commercial (CC BY-NC 4.0) license, which permits others to distribute, remix, adapt, build upon this work non-commercially, and license their derivative works on different terms, provided the original work is properly cited, appropriate credit is given, any changes made indicated, and the use is non-commercial. See: http://creativecommons.org/licenses/by-nc/4.0/.

\section{ORCID iDs}

Tanvi Khera http://orcid.org/0000-0002-0508-9409

Valerie M Banner-Goodspeed http://orcid.org/0000-0002-7644-2521

Lena Novack http://orcid.org/0000-0003-3748-2632

Balachundhar Subramaniam http://orcid.org/0000-0001-6510-3885

\section{REFERENCES}

1 Rudolph JL, Marcantonio ER. Review articles: postoperative delirium: acute change with long-term implications. Anesth Analg 2011;112:1202-11.

2 Saczynski JS, Marcantonio ER, Quach L, et al. Cognitive trajectories after postoperative delirium. N Engl J Med 2012;367:30-9.

3 Hovens IB, van Leeuwen BL, Mariani MA, et al. Postoperative cognitive dysfunction and neuroinflammation; cardiac surgery and abdominal surgery are not the same. Brain Behav Immun 2016;54:178-93.

4 Inouye SK, Marcantonio ER, Kosar CM, et al. The short-term and long-term relationship between delirium and cognitive trajectory in older surgical patients. Alzheimers Dement 2016;12:766-75.

5 Evered L, Silbert B, Knopman DS, et al. Recommendations for the nomenclature of cognitive change associated with anaesthesia and surgery-2018. Anesthesiology 2018;129:872-9.

6 Inouye SK, Westendorp RGJ, Saczynski JS. Delirium in elderly people. Lancet 2014;383:911-22.

7 Newman MF, Kirchner JL, Phillips-Bute B, et al. Longitudinal assessment of neurocognitive function after coronary-artery bypass surgery. N Engl J Med 2001;344:395-402.

8 Gosselt AN, Slooter AJ, Boere PR, et al. Risk factors for delirium after on-pump cardiac surgery: a systematic review. Crit Care 2015;19:346.

9 Bilotta F, Lauretta MP, Borozdina A, et al. Postoperative delirium: risk factors, diagnosis and perioperative care. Minerva Anestesiol 2013;79:1066-76.

10 van den Boogaard M, Kox M, Quinn KL, et al. Biomarkers associated with delirium in critically ill patients and their relation with long-term subjective cognitive dysfunction; indications for different pathways governing delirium in inflamed and noninflamed patients. Crit Care 2011;15:R297.

11 Bronicki RA, Hall M. Cardiopulmonary bypass-induced inflammatory response: pathophysiology and treatment. Pediatr Crit Care Med 2016;17:S272-8.

12 Djaiani G, Silverton N, Fedorko L, et al. Dexmedetomidine versus propofol sedation reduces delirium after cardiac surgery: a randomized controlled trial. Anesthesiology 2016;124:362-8.

13 Li X, Yang J, Nie X-L, et al. Impact of dexmedetomidine on the incidence of delirium in elderly patients after cardiac surgery: a randomized controlled trial. PLoS One 2017;12:e0170757.

14 Siddiqi N, Harrison JK, Clegg A, et al. Interventions for preventing delirium in hospitalised non-ICU patients. Cochrane Database Syst Rev;69.

15 Oh ES, Needham DM, Nikooie R, et al. Antipsychotics for preventing delirium in hospitalized adults: a systematic review. Ann Intern Med 2019;171:474-84.

16 Nikooie R, Neufeld KJ, Oh ES, et al. Antipsychotics for treating delirium in hospitalized adults: a systematic review. Ann Intern Med 2019;171:485-95. 
17 Bignami E, Castella A, Pota V, et al. Perioperative pain management in cardiac surgery: a systematic review. Minerva Anestesiol 2018;84:488-503.

18 de Souza Brito F, Mehta RH, Lopes RD, et al. Nonsteroidal antiinflammatory drugs and clinical outcomes in patients undergoing coronary artery bypass surgery. Am J Med 2017;130:462-8.

19 Wick EC, Grant MC, Wu CL. Postoperative multimodal analgesia pain management with nonopioid analgesics and techniques: a review. JAMA Surg 2017;152:691-7.

20 Gelman D, Gelmanas A, Urbanaite D, et al. Role of multimodal analgesia in the evolving enhanced recovery after surgery pathways. Medicina 2018;54:20.

21 Botting RM. Mechanism of action of acetaminophen: is there a cyclooxygenase 3? Clin Infect Dis 2000;31:S202-10.

22 Piletta P, Porchet HC, Dayer P. Central analgesic effect of acetaminophen but not of aspirin. Clin Pharmacol Ther 1991;49:350-4.

23 Singla NK, Parulan C, Samson R, et al. Plasma and cerebrospinal fluid pharmacokinetic parameters after single-dose administration of intravenous, oral, or rectal acetaminophen. Pain Pr 2012;12:523-32.

24 Jibril F, Sharaby S, Mohamed A, et al. Intravenous versus oral acetaminophen for pain: systematic review of current evidence to support clinical decision-making. Can J Hosp Pharm 2015;68:238-47.

25 Douzjian DJ, Kulik A, Drug O. Old drug, new route: a systematic review of intravenous acetaminophen after adult cardiac surgery. $J$ Cardiothorac Vasc Anesth 2017;31:694-701.

26 Graham GG, Scott KF. Mechanism of action of paracetamol. Am J Ther 2005; 12:46-55.

27 Subramaniam B, Shankar P, Shaefi S, et al. Effect of intravenous acetaminophen vs placebo combined with propofol or dexmedetomidine on postoperative delirium among older patients following cardiac surgery: the DEXACET randomized clinical trial. JAMA 2019;321:686-96.

28 Inouye SK, van Dyck CH, Alessi CA, et al. Clarifying confusion: the confusion assessment method. A new method for detection of delirium. Ann Intern Med 1990;113:941-8.

29 Julayanont P, Phillips N, Chertkow H. Montreal Cognitive Assessment (MoCA): Concept and Clinical Review. In: Larner AJ, ed. Cognitive screening instruments: a practical approach. London: Springer London, 2012: 111-51.

30 Almeida OP, Almeida SA. Short versions of the geriatric depression scale: a study of their validity for the diagnosis of a major depressive episode according to ICD-10 and DSM-IV. Int J Geriatr Psychiatry 1999;14:858-65.

31 Albert MS, Levkoff SE, Reilly C, et al. The delirium symptom interview: an interview for the detection of delirium symptoms in hospitalized patients. J Geriatr Psychiatry Neurol 1992;5:14-21.

32 Pfeffer RI, Kurosaki TT, Harrah $\mathrm{CH}$, et al. Measurement of functional activities in older adults in the community. J Gerontol 1982;37:323-9.

33 Gleason LJ, Benton EA, Alvarez-Nebreda ML, et al. FRAIL questionnaire screening tool and short-term outcomes in geriatric fracture patients. J Am Med Dir Assoc 2017;18:1082-6.

34 Ware J, Kosinski M, Keller SD. A 12-Item Short-Form health survey: construction of scales and preliminary tests of reliability and validity. Med Care 1996;34:220-33.

35 Ely EW, Margolin R, Francis J, et al. Evaluation of delirium in critically ill patients: validation of the Confusion Assessment Method for the intensive care unit (CAM-ICU). Crit Care Med 2001;29:1370-9.

36 Pendlebury ST, Welch SJV, Cuthbertson FC, et al. Telephone assessment of cognition after transient ischemic attack and stroke: modified telephone interview of cognitive status and telephone Montreal Cognitive Assessment versus face-to-face Montreal Cognitive Assessment and neuropsychological battery. Stroke 2013;44:227-9.

37 Vasunilashorn SM, Fong TG, Albuquerque A, et al. Delirium severity post-surgery and its relationship with long-term cognitive decline in a cohort of patients without dementia. $J$ Alzheimers Dis 2018;61:347-58

38 Hawker GA, Mian S, Kendzerska T, et al. Measures of adult pain: Visual Analog Scale for Pain (VAS Pain), Numeric Rating Scale for Pain (NRS Pain), McGill Pain Questionnaire (MPQ), Short-Form McGill Pain Questionnaire (SF-MPQ), Chronic Pain Grade Scale (CPGS), Short Form-36 Bodily Pain Scale (SF-36 BPS), and Measure of Intermittent and Constant Osteoarthritis Pain (ICOAP). Arthritis Care Res 2011;63:S240-52. 